\title{
Calidad de servicio en farmacias tradicionales y de autoservicio: Estudio de caso*
}

\author{
Castellano, Susie** \\ González, Pamela***
}

\section{Resumen}

La calidad de servicio hoy en día, debe representar una de las estrategias de toda empresa si pretende mantenerse dentro del mercado con altos niveles competitivos. El objetivo principal de la presente investigación es comparar la calidad del servicio de las farmacias con enfoque tradicional y de autoservicio del municipio Maracaibo. La metodología consiste en la revisión documental, fuentes secundarias y la aplicación de dos instrumentos para medir la variable, las cuales consisten en una lista de verificación a las farmacias con escala dicotómica para identificar sus características y una entrevista estructurada con escala de Likert a los usuarios de las farmacias. Los resultados arrojaron la existencia de una mediana calidad de servicio de las farmacias tradicionales y una alta calidad para las farmacias de autoservicio en el municipio Maracaibo del estado Zulia, por lo que se recomienda a las tradicionales enfocarse en sus puntos débiles para superarlos tomando en cuenta las fortalezas de las farmacias de autoservicio.

Palabras clave: Calidad del servicio, farmacias, dimensiones de la calidad de servicio, ServPerf.

Recibido: 09-03-10. Aceptado: 29-10-10

* Este artículo forma parte del proyecto "Calidad de servicio en las farmacias del Estado Zulia" adscrito al programa "Calidad de servicio en diferentes sectores de la Región Zuliana", de la Escuela de Ingeniería Industrial (LUZ), financiado por el Consejo de Desarrollo Científico, Humanístico y Tecnológico (CONDES) de la Universidad del Zulia (LUZ).

** Ingeniera Industrial (LUZ). Maestría en Gerencia de Empresas. Mención Gerencia de Mercadeo (LUZ). Profesora Agregada de la Facultad de Ingeniería de La Universidad del Zulia (LUZ). Profesora de la Facultad de Ingeniería de la Universidad Rafael Urdaneta (URU). Investigadora del Centro de Investigaciones de Ingeniería Industrial de la FACING de LUZ. e-mail: susiecg @cantv.net.

*** Ingeniera Industrial egresada de la Universidad del Zulia (LUZ), Auxiliar de Investigación del Centro de Investigaciones de Ingeniería Industrial de la FACING de LUZ. e-mail: pamediprizio@gmail.com. 


\section{Quality of Service in Traditional and Self-Service Pharmacies in the Maracaibo Municipality, Zulia State}

\section{Abstract}

Today, quality of service should be a strategy for every company if it intends to stay in the market at high competitive levels. The main objective of this research is to compare the quality of service for traditional pharmacies and the self-service approach in Maracaibo. Methodology consisted of documentary review, secondary sources and applying two instruments to measure the variable: a checklist for pharmacies with a dichotomous scale to identify their characteristics and a structured interview with a Likert scale for pharmacy users. Results showed the existence of a medium quality of service at traditional pharmacies and high quality at self-service pharmacies in the municipality of Maracaibo, State of Zulia. It is recommended that traditional pharmacies focus on overcoming their weaknesses, taking into account the strengths of self-service pharmacies.

Key words: Quality of service, pharmacy, dimensions of the quality of service, ServPerf.

\section{Introducción}

La competencia y la necesidad de satisfacer las expectativas de los clientes adquieren cada vez más importancia e imponen requerimientos cada vez más fuertes sobre las empresas. Tales expectativas abarcan no solo el precio y la calidad de los productos o servicios, sino también la rapidez, confiabilidad y eficiencia en la entrega. Todo esto es lo que se ha llamado "calidad de servicio", la cual se ha convertido en nuestros días en un requisito imprescindible para competir a nivel mundial, convirtiéndose en un elemento estratégico que confiere una ventaja competitiva en el tiempo a aquellas organizaciones que tratan de alcanzarla.

El servicio al cliente puede considerarse como un valor adicional en el caso de productos tangibles pero constituye la esencia de las empresas de servicios, como en este caso lo son las farmacias. Al ser el mismo un elemento más subjetivo y menos afectado por las nuevas tecnologías, el cliente aplica su capacidad crítica con mayor intensidad a los servicios que recibe que a los productos que consume. Por otro lado, medir la calidad de servicio resulta más difícil y complicado que poder apreciar la perfección de un producto, ya que el servicio lo brindan las personas y se percibe de una forma mucho más subjetiva. Un factor determinante es la actitud del personal que proporciona los servicios y promueve la venta de productos de la empresa, ya que la percepción de un producto puede mejorar con la presentación de los bienes comerciales que ofrecen los vendedores a los consumidores.

Franco (2002:1), presidenta de Factor Humano, empresa consultora en procesos de calidad, señala "La percepción que tienen los clientes externos de la calidad de la atención que reciben de una compañía depende directamente de la calidad del servicio que ésta posea inter- 
namente. En consecuencia, la prioridad de una organización que desea alcanzar niveles superiores en calidad de servicio, debe estar focalizada en la creación y reforzamiento de una cultura cuya base sea la satisfacción de su propio recurso humano".

El rubro de la salud es uno de los que más incide en la actividad económica de nuestro país. Por esta razón, las farmacias han tenido que cambiar su estrategia de supervivencia, mostrando en el negocio una eficiencia en la estructura de costos, multiplicidad de ofertas para atrapar a los consumidores, disponibilidad para trabajar corrido y más de las ocho horas reglamentarias, mantener inventarios bajos de alta rotación y el ofrecimiento de servicios y productos conexos a la actividad farmacéutica (Orjuela, 2003).

Debido al crecimiento de las cadenas farmacéuticas y la alta demanda de calidad de servicio por parte de los clientes, el objetivo principal de esta investigación es comparar la calidad de servicio que ofrecen las farmacias con enfoque tradicional (aquellas donde solo se expenden medicamentos y el cliente es atendido por una persona detrás de un mostrador) y la ofrecida por las de autoservicio (donde se consigue mucho más que medicinas y existen anaqueles donde el cliente puede servirse de lo que necesita) en el municipio Maracaibo, ya que se presume que existen factores dentro del servicio brindado que las diferencian a ambas debido a sus distintas gestiones de venta, además de dar a conocer de qué manera se orientan a brindar una mejor atención al cliente y cómo es la calidad de servicio percibida por estos últimos.

\section{Significado de la calidad de servicio}

La calidad de servicio, de acuerdo con Berry (1992), es la percepción de un cliente acerca de la correspondencia entre el desempeño y las expectativas, relacionados con el conjunto de elementos secundarios, cuantitativos, cualitativos, de un producto o servicio principal. Es formulado a la luz de la percepción del consumidor y sólo puede definirse con base en las especificaciones que satisfagan sus necesidades, Por lo tanto, es el consumidor quien determina lo que es la calidad (Cobra, 2000).

Horovitz (1994) afirma que la misma es responsabilidad de toda la organización; empleados, gerentes y todas aquellas personas que están en contacto con la clientela, deben participar unidos en una amplia búsqueda organizada de errores, que sólo conseguirá llegar a cero defectos, si todos la suscriben con el mismo entusiasmo. Asimismo, desde la óptica de las percepciones de los clientes, puede ser definida como la amplitud de la discrepancia o diferencia que exista entre las expectativas o deseos de los clientes y sus percepciones.

Por lo tanto, la calidad del servicio está representada por el grupo de características que definen las organizaciones sobre acciones intangibles que ofrece a sus clientes, de acuerdo a sus necesidades y expectativas, quien las evalúa y cataloga como satisfactorias o no de acuerdo a su percepción.

Para complementar el concepto, es necesario hacer referencia a conceptos como necesidades, expectativas y percepciones del cliente. 


\subsection{Necesidades del cliente}

Las necesidades vienen a representar una carencia que posee el individuo sobre algún producto, servicio o bienestar fisiológico que lo conlleva a la constante búsqueda de satisfacerla.

Para respaldar tal aseveración, Zeithaml y Bitner (2002), explican que las necesidades son condiciones fundamentales para el logro del bienestar físico o fisiológico del cliente y que dan forma al nivel de servicio deseado. Son la fuerza básica que impulsa al ser humano a realizar algo (McCarthy y Perreault, 1999). Las necesidades son dinámicas, cambiando constantemente en el tiempo, por lo que no debe pensarse que se conocen suficientemente bien (Cobra 2000).

\subsection{Expectativas del cliente}

McCarthy y Perreault (1999), plantean que las expectativas son la combinación de las creencias y actitudes del consumidor. Es el resultado de algo que se espera que se realice. Se fundamentan en experiencias vividas por el cliente al comprar, en opiniones de otras personas y en la información recibida y promesas del comercializador y de la competencia (Kotler y Armstrong, 1998).

Agrega Fernández (2000), que los clientes que intentan comprar un producto o recibir un servicio, por lo general no desean ningún producto o servicio determinado, sino satisfacer una expectativa.

En líneas generales, es lo que espera recibir una persona de un producto 0 servicio que adquiere, en función de un patrón prefijado previamente.

\subsection{Percepción}

La percepción, según Kotler y Armstrong (1998), es el proceso mediante el cual las personas seleccionan, organizan e interpretan la información, para formar una imagen significativa del mundo. Es la manera como los individuos adquieren e interpretan la información del ambiente que les rodea (McCarthy y Perreault, 1999).

En este sentido, viene a ser la interpretación que hace una persona acerca de algún estímulo que recibe de su entorno mediante sus sentidos. Cuando una persona recibe un servicio, éste lo califica de bueno o malo, según la impresión que tenga al momento de recibirlo en función del trato recibido de parte del prestador del servicio.

\section{Dimensiones de la calidad de servicio}

Existe diversidad de teorías acerca de las dimensiones que se deben tomar en cuenta a la hora de medir la calidad de servicio. Para este estudio se tomó en cuenta el planteamiento realizado por $\mathrm{Pa}$ rasuraman y sus colaboradores (1991), los cuales lograron determinar que, cuando el cliente percibe el servicio que recibe, básicamente lo hace tomando en cuenta diferentes criterios, entre los cuales se mencionan:

- Tangibilidad o elementos tangibles: aquellos elementos físicos que van acompañados con la prestación del servicio, se refiere a la apariencia de las instalaciones físicas, equipos, material de comunicación y personal, 
de los cuales el cliente podría eventualmente emitir un juicio parcial acerca de la calidad del servicio de la empresa.

- Confiabilidad: habilidad que posee la empresa para suministrar el servicio en el momento preciso, cuando sea requerido y de manera correcta.

- Capacidad de respuesta: actitud que muestra la organización en el momento de ofrecer soluciones a los clientes y proporcionarles servicios rápidos; dependerá del cumplimiento a tiempo del compromiso contraído con el cliente y también de qué tan accesible es entrar en contacto con la compañía. En pocas palabras, disposición y voluntad para ayudar a los usuarios y proporcionar un servicio rápido.

- Seguridad: Esta dimensión consiste en la credibilidad que poseen los clientes de cómo las empresas solucionan sus problemas y el interés demostrados por ésta para satisfacer sus necesidades. Los factores a considerar por una organización para transmitir seguridad son: crear y mantener una buena reputación del nombre de la compañía, capacitar al personal que está en contacto con el cliente, transmitir confianza y evitar cualquier interacción agresiva con el mismo.

- Empatía: atención individualizada, amable, proporcionada por la empresa a sus clientes, en otras palabras, se refiere al contacto directo entre la organización y el cliente. Para que la empatía ocurra debe necesariamente existir una buena comunicación entre la empresa y el cliente, informándolo de todos aquellos aspectos del servicio.

\section{Modelos de evaluación de la calidad de servicio}

Los modelos más reconocidos proponen que la calidad que se percibe de un servicio es el resultado de una comparación entre las expectativas del cliente y las cualidades del servicio. Dos de los modelos que se utilizan para evaluar la variable son: el modelo SERVQUAL y el SERVPERF.

\subsection{Modelo SERVQUAL}

EI SERVQUAL es un instrumento, en forma de cuestionario, elaborado por, Parasuraman, Zeithaml y Berry cuyo propósito es evaluar la calidad de servicio ofrecida por una organización a lo largo de cinco dimensiones: fiabilidad, capacidad de respuesta, seguridad, empatía y elementos tangibles. Está constituido por una escala de respuestas múltiples, diseñada para comprender las expectativas de los clientes respecto a un servicio. Permite evaluar, pero también es un instrumento de mejora y de comparación con otras organizaciones (Parasuraman et al., 1991).

El cuestionario comprende dos partes: la primera compuesta por 22 afirmaciones que tratan de identificar las expectativas generales de los clientes sobre un servicio concreto. Y la segunda dedicada a las percepciones, formada por las mismas 22 afirmaciones anteriores, donde la única diferencia es que no hacen referencia a un servicio específico 
sino a lo que reciben de una empresa que ofrece tal servicio.

\subsection{Modelo SERVPERF}

Cronin y Taylor (Setó, 2004), proponen el modelo alternativo, el SERVPERF, basado en el desempeño, donde se mide solamente las percepciones que tienen los consumidores acerca del rendimiento del servicio, para lo cual se emplea el mismo instrumento utilizado en el modelo SERVQUAL, pero descartando la identificación de las expectativas. Los autores afirman que dicha escala basada solo en las percepciones o en el resultado real mide mejor la calidad del servicio. Consideran además, que la calidad de un servicio debe ser medida como una actitud, es decir, sólo a través de la percepción y no comparando las expectativas con la percepción. La calidad del servicio será por tanto mejor, cuanto mayor sea la suma de dichas percepciones. La escala SERVPERF produce como resultado un puntaje acumulado de la calidad general de un servicio, lo cual puede representarse mediante una gráfica relativa al tiempo y a subgrupos específicos de consumidores. El modelo emplea los veintidós (22) puntos identificados por el método SERVQUAL, simplificando por tanto a la mitad las mediciones con respecto al SERVQUAL. De esta manera, el modelo SERVPERF conforma las implicaciones teóricas sobre las actitudes y satisfacción. En el Cuadro 1 se encuentran los ítems que se estudian.

En conclusión, la escala es la misma, lo que varía es el enfoque de evaluación y las preguntas en los instrumentos. Otra conclusión importante es que no existe un instrumento de medición de la calidad universal para todos los servicios, sino que en una industria, el cuestionario y las dimensiones de la calidad del servicio serán diferentes a otra. Esta escala intenta superar las limitaciones de utilizar las expectativas en la medición de la calidad percibida, sin definir concretamente el tipo y el nivel de expectativas a utilizar (expectativas de desempeño, experienciales, predictivas, normativas, entre otros).

\section{Modelo propuesto de medición de la calidad del servicio de las farmacias}

A fin de alcanzar un valor un tanto objetivo de la calificación que se obtienea través de entrevistas realizadas a los usuarios de farmacias, la investigación se enfoca en obtener un híbrido al unir la llamada "Calidad Objetiva" con la "Calidad Percibida".

Se define entonces la calidad objetiva, real o mecánica, como aquella que se desprende de la cuantificación y comprobación de la superioridad de un estándar que se considera medible; se relaciona con la descripción de la excelencia de los productos (Zeithaml, 1988, citado por Hernández et al., 2006).

La calidad subjetiva, está relacionada con la apreciación del consumidor acerca de ciertos parámetros determinados del servicio. Robledo (2004) asevera que cuando la calidad es definida desde el punto de vista del consumidor, éste adquiere un matiz subjetivo, idiosincrático, ya que se trata de una evaluación percibida y por tanto, una forma personal de interpretar y dar sentido a estímulos externos. 


\section{Cuadro 1 \\ Ítems del Modelo ServQual y ServPerf}

Dimensión 1: Elementos tangibles

- La empresa de servicios tiene equipos de apariencia moderna.

- Las instalaciones físicas de la empresa de servicios son visualmente atractivas.

- Los empleados de la empresa de servicios tienen apariencia pulcra.

- Los elementos materiales (folletos, estados de cuenta y similares) son visualmente atractivos.

Dimensión 2: Fiabilidad

- Cuando la empresa de servicio promete hacer algo en cierto tiempo lo hace.

- Cuando un cliente tiene un problema la empresa muestra un sincero interés en solucionarlo.

- La empresa realiza bien el servicio la primera vez.

- La empresa concluye el servicio en el tiempo prometido.

- La empresa de servicios insiste en mantener registros exentos de errores.

Dimensión 3: Capacidad de respuesta

- Los empleados comunican a los clientes cuando concluirá la realización del servicio.

- Los empleados de la empresa ofrecen un servicio rápido a sus clientes.

- Los empleados de la empresa de servicios siempre están dispuestos a ayudar a sus clientes.

- Los empleados nunca están demasiado ocupados para responder a las preguntas de sus clientes.

\section{Dimensión 4: Seguridad}

- El comportamiento de los empleados de la empresa de servicios transmite confianza a sus clientes.

- Los clientes se sienten seguros en sus transacciones con la empresa de servicios.

- Los empleados de la empresa de servicios son siempre amables con los clientes.

- Los empleados tienen conocimientos suficientes para responder a las preguntas de los clientes.

\section{Dimensión 5: Empatía}

- La empresa de servicios da a sus clientes una atención individualizada.

- La empresa de servicios tiene horarios de trabajo convenientes para todos sus clientes.

- La empresa de servicios tiene empleados que ofrecen una atención personalizada a sus clientes.

- La empresa de servicios se preocupa por los mejores intereses de sus clientes.

- La empresa de servicios comprende las necesidades específicas de sus clientes.

Fuente: Parasuraman et al. (1991).

Basados en estos conceptos, el modelo de medición de la calidad de servicio que se propone se fundamentó en los siguientes aspectos:

- Para la calidad objetiva, se toman en cuenta las características físicas que poseen las farmacias como elementos tangibles de la calidad de servicio, es decir, todos aquellos aspectos que pueden ser evaluados objetivamente mediante la observación directa y todo lo que deben poseer las 
farmacias, que en muchos casos es desconocido por los usuarios, limitándolo a proporcionar un juicio objetivo. Para esto, se utilizó una lista de verificación aplicada por las investigadoras, donde se visitó cada farmacia seleccionada al azar y se constató la existencia o no de los parámetros considerados.

- Para medir la calidad percibida, se aplica un instrumento adaptado del modelo ServPerf y de la revisión literaria sobre establecimientos de farmacia. Dicho instrumento, mide la percepción del servicio por parte de los usuarios de las farmacias, tomando en cuenta las dimensiones del mo- delo ServQual, relacionadas con la calidad de servicio.

\section{Resultados del estudio}

Para el estudio, se emplearon las mismas dimensiones definidas con anterioridad en el modelo SERVQUAL y SERVPERF, y se diseñaron una serie de indicadores que permiten medir la calidad de servicio. En el Cuadro 2 se pueden observar dichos indicadores con sus dimensiones correspondientes.

Es importante resaltar, que las farmacias representan un rubro muy importante dentro del sector salud con demanda creciente, es por ello que se hace im-

\section{Cuadro 2}

\section{Dimensiones e indicadores de la calidad del servicio de las farmacias}

\begin{tabular}{|c|c|}
\hline Dimensiones & Indicadores \\
\hline Elementos tangibles & $\begin{array}{l}\text { - Personal. } \\
\text { - Orden y limpieza. } \\
\text { - Iluminación. } \\
\text { - Condiciones generales. } \\
\text { - Equipos utilizados. } \\
\text { - Almacenamiento }\end{array}$ \\
\hline Fiabilidad & $\begin{array}{l}\text { - Consejo farmacéutico. } \\
\text { - Precio. } \\
\text { - Servicio prometido. }\end{array}$ \\
\hline Seguridad & $\begin{array}{l}\text { - Trato de los empleados. } \\
\text { - Confianza. } \\
\text { - Organización de productos. }\end{array}$ \\
\hline Empatía & $\begin{array}{l}\text { - Atención personalizada. } \\
\text { - Horarios. } \\
\text { - Comunicación. } \\
\text { - Actividades extras. } \\
\text { - Servicios adicionales. }\end{array}$ \\
\hline Capacidad de respuesta & $\begin{array}{l}\text { - Tiempo de respuesta. } \\
\text { - Mejora continua. }\end{array}$ \\
\hline
\end{tabular}

Fuente: Elaboración propia. 
portante hacer notar las particularidades que diferencian una farmacia de otra, y los servicios claves que éstas prestan. En el Cuadro 3 se pueden observar algunas características y servicios en ambos tipos de farmacia.

Para efectos de medir la calidad de servicio en las farmacias tradicionales y de autoservicio del municipio Maracaibo, se utilizó un muestreo aleatorio estratificado para la población constituida por las características presentes en ambas modalidades de farmacia, utilizando para esto un "check list" aplicado por las investigadoras, y estudiándose 61 farmacias tradicionales y 9 farmacias de autoservicio. Para la segunda población, constituida por la percepción de los usuarios acerca de la calidad de servicio de las farmacias, se utilizó un muestreo por conglomerados. Se aprovechó la visita realiza- da a cada una de las farmacias para seleccionar los conglomerados (dentro de un horario de 12 horas de trabajo del establecimiento), los cuales quedaron conformados por todos los clientes que visitaron dicha farmacia en el lapso de una (1) hora. Se utilizó un instrumento escala de Likert aplicado a los usuarios.

La escala de medición empleada para evaluar la calidad del servicio en las farmacias tradicionales y de autoservicio se muestra en la Tabla 1.

Los resultados arrojados por las encuestas aplicadas indican la existencia de diferencias en cuanto a la calidad del servicio prestado en ambas farmacias. Tales diferencias se ampliarán mediante la comparación de sus dimensiones e indicadores a través de los resultados obtenidos, para finalmente dar a conocer la evaluación.

\section{Cuadro 3 \\ Características y servicios de las farmacias Tradicionales y de Autoservicio}

Farmacias Tradicionales

- Amplio stock de productos farmacéuticos

- Asesoría farmacéutica

- Preparaciones Magistrales

- Productos de cuidado personal

- Artículos para bebé, productos naturales

- Material médico quirúrgico y misceláneas

- Estacionamiento reducido

- Horario de trabajo limitado por turnos
Farmacias de Autoservicio

- Establecimientos amplios

- Amplio stock de productos farmacéuticos y misceláneos

- Preparaciones magistrales

- Asesoría farmacéutica personalizada

- Amplia gamma de productos de cuidado personal, golosinas, artículos para el hogar, bebidas gaseosas, bebidas energizantes, víveres, productos fotográficos

- Equipos para medición de tensión, frecuencia cardíaca, pesos y otros parámetros de salud

- Leche maternizada, colados y artículos para bebé

- Productos naturales

- Amplio estacionamiento para los clientes

- Horario de trabajo: 365 días del año y las 24 horas al día

Fuente: Elaboración propia. Observaciones directas hechas a las farmacias. 


\section{Tabla 1}

Escala de medición de la calidad del servicio en las farmacias

\begin{tabular}{lc}
\hline \multicolumn{1}{c}{ Escala } & Valor \\
\hline No existe calidad de servicio & $1,00-1,80$ \\
Baja calidad de servicio & $1,81-2,60$ \\
Mediana calidad de servicio & $2,61-3,40$ \\
Buena calidad de servicio & $3,41-4,20$ \\
Alta calidad de servicio & $4,21-5,00$ \\
\hline
\end{tabular}

Fuente: Elaboración propia.

\subsection{Dimensión: Elementos Tangibles}

En líneas generales, las farmacias de autoservicio son evaluadas por sus clientes como empresas que le dan importancia a sus elementos tangibles (elementos físicos que van acompañados con la prestación del servicio), mientras que para las farmacias tradicionales no es algo primordial a la hora de prestar sus servicios al cliente. Los elementos tangibles son un factor preponderante en lo que se refiere al aumento de la percepción de la calidad del servicio, las farmacias al concentrarse en los elementos tangibles están mejorando la manera en la cual el cliente valora la misma por medio del sentido de la vista; el ubicar las tiendas en sitios de alto tránsito tanto peatonal como vehicular, ofrecer a los clientes amplios estacionamientos, poseer servicio de vigilancia, acceso a los productos por parte de los clientes (algunas tiendas de autoservicio tienen estándares en los cuales todas sus tiendas están distribuidas de la misma manera lo cual ayuda al cliente a encontrar el producto que busca fácilmente en cualquier sucursal), amplios pasillos permitiendo desplazarse con carritos o cestas de compra con comodidad, aire acondicionado, iluminación estratégicamente diseñada para realzar la imagen de la tienda y de los productos, son factores que sin lugar a duda atraen al cliente. Es más común observar este tipo de características en las farmacias de autoservicio que en las tradicionales, razón por la cual las de autoservicio son valoradas por encima de las farmacias tradicionales en cuanto a sus elementos tangibles.

En el Gráfico 1 se muestra la comparación de las farmacias tradicionales y las de autoservicio en cuanto a la dimensión Elementos Tangibles y los indicadores asociados.

La diferencia más significativa en cuanto a esta dimensión se manifiesta en el indicador iluminación. Puede interpretarse que los clientes aprecian que las farmacias de autoservicio tengan una mayor y mejor cantidad y calidad de iluminación (en cuanto a luz artificial y natural se refiere) que las farmacias tradicionales. Según Monroy (2006) un buen alumbrado actúa como un vendedor silencioso, ayudando al futuro cliente a dirigir su atención hacia determinados artículos; además la buena iluminación garantiza el tránsito por el interior de los locales, con- 


\section{Gráfico 1 \\ Indicadores de la Calidad de Servicio para la dimensión Elementos Tangibles}

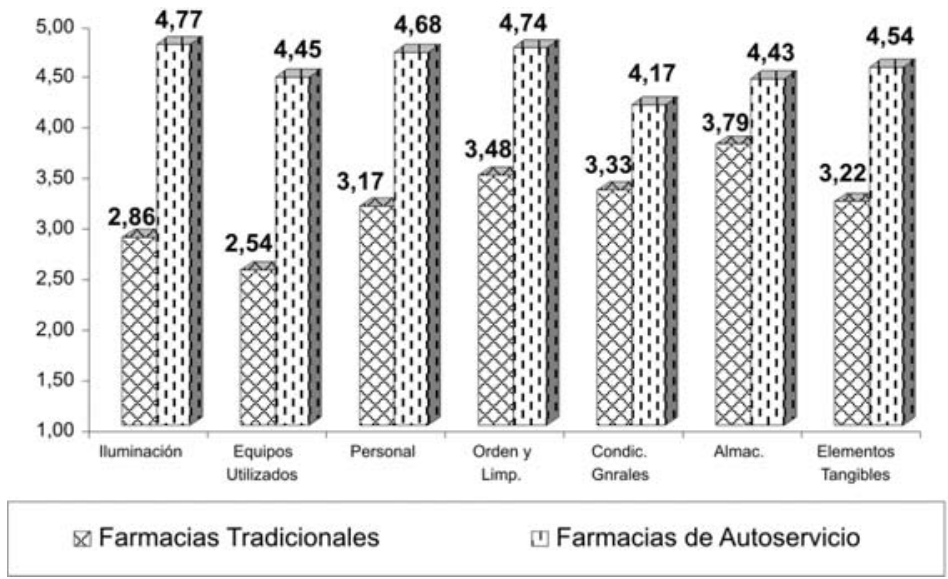

Fuente: Elaboración propia. Datos obtenidos a partir de las encuestas aplicadas (2009).

tribuyendo a la seguridad de los usuarios y a su vez un local comercial correctamente iluminado, se convierte en un lugar de trabajo confortable y por lo tanto agradable, contribuyendo a potenciar la actividad comercial.

De la misma manera, otro indicador que destaca por su alta diferencia entre los dos tipos de farmacia es el de equipos utilizados. Las farmacias tradicionales no presentan modernidad en sus equipos mientras que las farmacias de autoservicio utilizan equipos modernos y tecnológicamente a la vanguardia, diferencia que se basa principalmente en el auge reciente del mercado de las farmacias de autoservicio en nuestro país, además de las altas inversiones hechas por parte de las cadenas de farmacias o inversionistas para la instalación de cada tienda (Orjuela, 2003). La importancia de la utilización de equipos modernos se traduce en el he- cho de mejorar la productividad y agilizar tramitación de la documentación que se genera a lo largo del proceso productivo y administrativo de una farmacia (Bardera, 2008).

El indicador personal también es un factor con diferencia significativa. Las farmacias tradicionales pocas veces prestan atención a este indicador, mientras que las de autoservicio se ubican como empresas que dan una alta importancia a factores como la apariencia física del personal, aseo personal, vestimenta y cantidad de personal laborando, ya que estos son elementos determinantes en la atención al cliente, la uniformidad en la vestimenta y lo bien arreglado que esté el personal, es primordial, ya que constituye la cara del servicio. Soportando estos comentarios, se plantea lo expuesto por Romero (2006), donde los modales y el uso del uniforme como vestuario corporativo, 
son características esenciales para crear las estrategias tangibles en los mensajes del servicio y ayudan a aumentar de manera indirecta la calidad del servicio que se brinde.

En cuanto al orden y limpieza, las farmacias de autoservicio están mejor posicionadas que las farmacias tradicionales; es importante señalar el valor de este indicador dentro de las farmacias, ya que parte de la calidad del servicio que el cliente percibe, está íntimamente relacionada con la apariencia física del local donde se desarrolla dicha actividad, a su vez, un local poco limpio y poco arreglado pone de manifiesto otros problemas que se escapan de la vista del cliente.

En cuanto a los indicadores restantes, estos no mostraron diferencias marcadas significativas; sin embargo, es necesario resaltar que en cuanto a las condiciones generales y el almacenamiento, las farmacias de autoservicio siguen estando a la vanguardia y por encima de las farmacias tradicionales tal como lo manifiestan los usuarios en las encuestas aplicadas.

\subsection{Dimensión Fiabilidad}

Tanto las farmacias tradicionales como las de autoservicio son evaluadas por sus clientes igualitariamente como empresas fiablemente "Buenas", en cuanto a la seguridad que debe ofrecer la empresa a sus clientes en suministrarle el servicio en el momento preciso, cuando sea requerido y de manera correcta. Elementos como el consejo farmacéutico, el precio y el servicio prometido son factores que inciden en el cliente para escoger la farmacia que visitará para comprar lo que en ese momento es una necesidad para él. Si el cliente necesita que le receten algún medicamento, con certeza acudirá a una donde hayan farmaceutas o personal capacitado para recetar medicamentos; si lo que necesita es economizar, buscará aquella cuyos descuentos y precios sean mejores, o si encontró alguna publicidad interesante, espera que las promesas dadas por medio de la publicidad sean cumplidas.

El Gráfico 2 muestra la comparación de las farmacias tradicionales y las de autoservicio en la dimensión Fiabilidad.

La diferencia más marcada en cuanto a los indicadores de la dimensión fiabilidad, se observa en el indicador consejo farmacéutico. Para los clientes entrevistados las farmacias tradicionales ofrecen un consejo farmacéutico aceptable, mientras que las de autoservicio se esmeran para brindar el mejor consejo farmacéutico proporcionando un servicio excelente y por encima de las farmacias tradicionales. Explicando este indicador se puede notar la presencia permanente de farmacéuticos especializados en las farmacias de autoservicio, los cuales, para los clientes son seguros y confiables a la hora de recomendar medicamentos y de brindar información importante cuando se le requiere.

En cuanto al precio no existe una diferencia notoria. Sin embargo, se hace importante resaltarlo ya que es el único indicador donde las farmacias tradicionales se destacan como mayores en contraposición a las de autoservicio, aunque la diferencia no es tan alta (menos del 1\%), se pone de manifiesto que, aunque las farmacias de autoservicio superan en muchos elementos a las tradicionales, 


\section{Gráfico 2 \\ Indicadores de la Calidad de Servicio para la dimensión Fiabilidad}

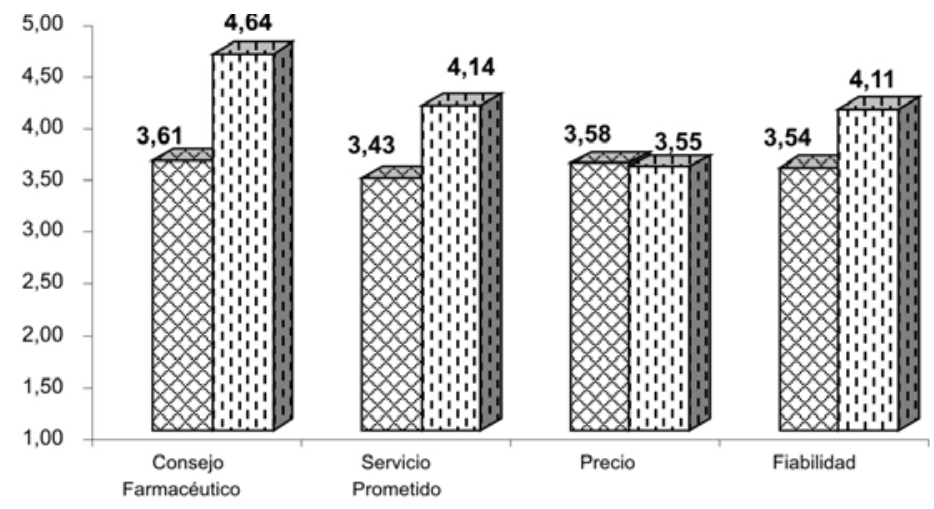

叉 Farmacias Tradicionales

Farmacias de Autoservicio

Fuente: Elaboración propia. Datos obtenidos a partir de las encuestas aplicadas (2009).

éstas últimas han logrado competir por medio de descuentos y brindando precios más bajos que la competencia, logrando con esto su permanencia dentro del mercado.

Tanto las farmacias tradicionales como las de autoservicio cumplen de manera satisfactoria o buena con el servicio prometido, tanto con los descuentos y promociones como con la ayuda que brindan a sus clientes cuando presentan algún problema.

\subsection{Dimensión Seguridad}

Las farmacias de autoservicio son evaluadas por sus clientes como empresas que superan en cuanto a la seguridad a las farmacias tradicionales. La seguridad en este caso, radica en el sentimiento que se genera en el cliente cuando coloca sus necesidades en manos del personal y de la farmacia como tal, confiando que estas necesidades serán resueltas de la mejor manera posible. Asimismo, se refiere a la competencia y cortesía del personal de servicio que infunde confianza en el cliente. Implica credibilidad, integridad, confiabilidad y honestidad. Lo que quiere decir que no solo es importante el cuidado de los intereses del cliente, sino que también la farmacia debe demostrar su preocupación en este sentido para brindarle al cliente una mayor satisfacción. Disponibilidad de productos, trato recibido por parte del empleado y disponibilidad de farmaceutas, son indicadores que elevan significativamente el nivel de calidad entre una farmacia y otra, además de elevar o disminuir el número de clientes y por ende sus ventas.

En el Gráfico 3 se visualizan los resultados de la "Seguridad" en ambos tipos de farmacia.

La diferencia más resaltante en la dimensión seguridad se observa en el in- 


\section{Gráfico 3 \\ Indicadores de la Calidad de Servicio para la dimensión Seguridad}

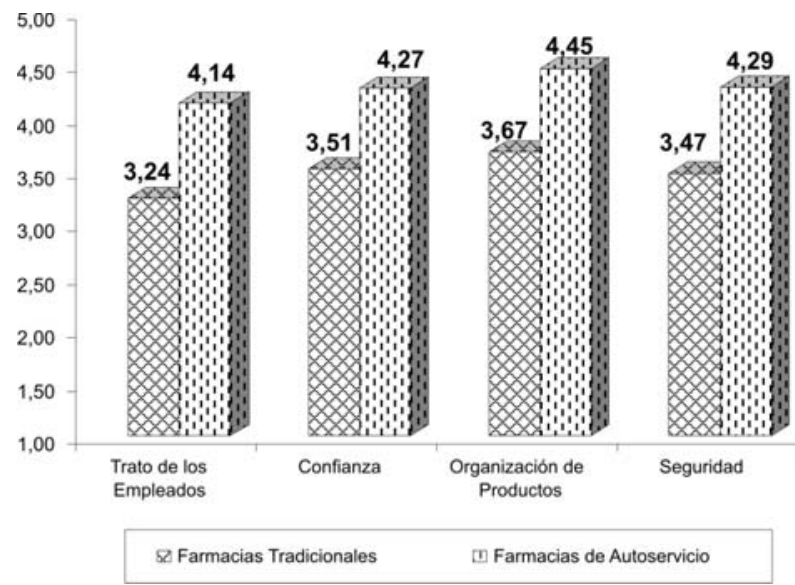

Fuente: Elaboración propia. Datos obtenidos a partir de las encuestas aplicadas (2009).

dicador trato de los empleados, los clientes muy pocas veces recomiendan las farmacias tradicionales a sus conocidos (a no ser que estén cerca) ya que los empleados les brindan un trato considerado "normal" mientras que en las de autoservicio, el trato dependiendo del horario y la cantidad de personas que estén en la farmacia, es un poco más "exclusivo". De esta forma las farmacias de autoservicio siguen superando a las tradicionales. Según Díaz (2008), un cliente que está conforme con la atención, será capaz de contarle a su círculo de amistades y familiares, la experiencia que tuvo con la farmacia. Un cliente feliz recomienda la farmacia y lo mejor es que para la empresa la publicidad es gratuita; basta solo con brindar un buen trato al cliente.

En cuanto al indicador confianza, las farmacias tradicionales están por debajo de las farmacias de autoservicio, específicamente en la disponibilidad de far- macéuticos en cualquier momento. En la organización de los productos, a pesar de la diferencia existente, los clientes expresaron en los dos tipos de farmacia no fijarse en eso, y señalaron además no haber tenido problemas con los medicamentos comprados.

\subsection{Dimensión Empatía}

Una farmacia se puede decir que es empática cuando ofrece atención personalizada, incluyendo el contacto organización-cliente, mantiene a los clientes informados y utiliza un lenguaje acorde a ellos, trabaja en horarios convenientes para los clientes, además de proporcionar actividades y servicios extras que la hacen sobresalir; poniéndose de esta manera en el lugar del cliente, identificando lo que siente, entendiendo lo que necesita y ofreciendo soluciones a sus problemas. De acuerdo a los resultados de la 
investigación, las farmacias de autoservicio son evaluadas por sus clientes como empresas empáticas mientras que las farmacias tradicionales son ubicadas por sus clientes en el rango de mediana calidad en cuando a esta dimensión. La amabilidad, discreción y sencillez son características que dejan una buena impresión en el consumidor. El respeto y la consideración hacia el cliente son básicos en las farmacias a la hora de medir la empatía.

El Gráfico 4 muestra la comparación de las farmacias tradicionales y las de autoservicio en la dimensión Empatía.

La diferencia más alta al comparar la empatía en las farmacias tradicionales y las de autoservicio se encuentra en el indicador actividades extras, que incluye realización de jornadas de educación y cuidado de la salud, expedición de cartas médicas e incluso facilidad para el pago de impuestos municipales, entre otros. Las farmacias tradicionales casi nunca realizan este tipo de acciones; en cambio, estas actividades extras son realizadas cotidianamente por las farmacias de autoservicio.

En cuanto al indicador servicios adicionales, las farmacias tradicionales están por debajo de las farmacias de autoservicio. Es importante hacer referencia que actualmente no existe gran diferencia entre las farmacias en cuanto a la aceptación de diversas formas de pago, ya que las farmacias tradicionales han tenido que ir diversificando su formato de ventas para poder seguir compitiendo en el mercado. Sin embargo, la diferencia existente la arroja el ítem concerniente a la prestación de servicios como: medición de la tensión, elaboración de exámenes clínicos, colocación de inyecciones, entre otros, servicios estos, que la mayoría de las veces son inexistentes en las farmacias tradicionales, mientras que en las de autoservicio si están disponibles.

Otro indicador importante es la comunicación, del cual se observa que exis-

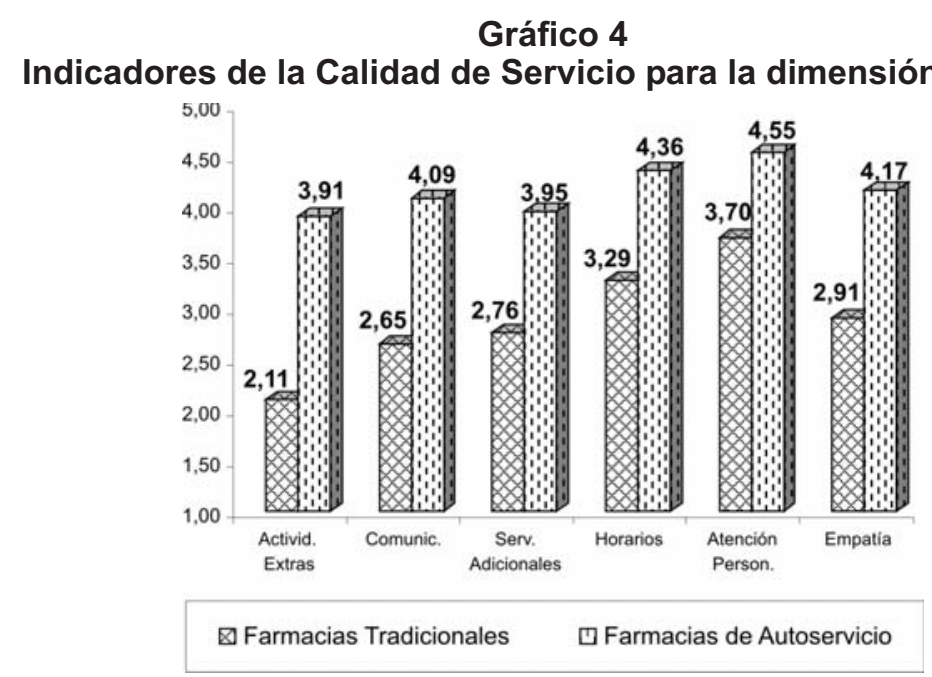

Fuente: Elaboración propia. Datos obtenidos a partir de las encuestas aplicadas (2009). 
te una diferencia marcada entre ellas; las farmacias de autoservicio emplean mejores medios para comunicar su información y hacerla llegar a sus clientes que las farmacias tradicionales, quienes muy pocas veces manejan este tipo de métodos para hacerle conocer al cliente sus promociones.

En cuanto a los horarios de trabajo, las farmacias de autoservicio se destacan ya que exceptuando una de ellas y aquellas ubicadas en centros comerciales, las mismas trabajan 24 horas todos los días; sin embargo, las farmacias tradicionales se rigen por el famoso "turno", el cual establece las que deben trabajar en forma rotativa las 24 horas y las que no, lo que las hace menos accesibles para los consumidores en comparación a las farmacias de autoservicio.

La atención personalizada es importante reseñarla también, ya que toda persona demandante de un servicio desea atención personalizada: espera ser tratada como si fuera el "único cliente", por medio de un trato cortés y respetuoso (Horovitz, 1994). Pese a que las farmacias de autoservicio son establecimientos donde el cliente debe por sí solo servirse de lo que necesita tomándolo de los estantes $\mathrm{o}$ anaqueles, éstas presentan ventajas, ya que el indicador está muy ligado con otros como lo son el consejo farmacéutico y los servicios adicionales. Al recibir el cliente un consejo o ayuda, siente que es atendido personalmente, porque es servido con lo que necesita, elevando la atención personal al punto de que algunas farmacias de autoservicio llevan el seguimiento de los tratamientos que sus clientes cumplen. Dentro de las misiones de las farmacias de autoservicio se en- cuentra el "trato personal o personalizado al cliente". Zubillaga (2008), al emitir su opinión específicamente acerca de una de las farmacias de autoservicio de la investigación, afirma que "hemos sabido crecer en el país, respetando siempre la premisa de mantener el contacto directo con el consumidor y sus necesidades $y$, dar un trato personal a la clientela". Por otra parte, Wharton (2005) expresa de las farmacias tradicionales que si quieren sobrevivir deberán especializarse en una atención más personalizada, disponibilidad rápida de mercadería y "delivery"; evidenciándose por medio de estos dos comentarios, una fortaleza de las farmacias de autoservicio respecto a las tradicionales en este aspecto.

\subsection{Dimensión Capacidad de Respuesta}

La capacidad de respuesta de una organización es también de suma importancia para el cliente. La rapidez con que el cliente es atendido, incide en la importancia que le transmite la organización a éste y será un severo crítico del servicio prestado. El mejoramiento del servicio es otro factor relevante para el cliente, como por ejemplo, si en la farmacia comienzan a automatizar los procesos de venta para facilitar la realización de las facturas, el cliente sentirá que de alguna manera el costo de los productos y servicios son retribuidos, ya que día a día el servicio está cambiando de manera positiva y en beneficio de él y no dudará en asistir de nuevo a la farmacia cuando presente alguna necesidad relacionada. La capacidad de respuesta dada a los clientes en las farmacias de autoservicio supera a la pro- 
porcionada por las farmacias tradicionales y esto puede visualizarse en el Gráfico 5 .

Mejora continua es el indicador que posee la diferencia más relevante; las farmacias tradicionales casi nunca mejoran su servicio, mientras que las de autoservicio lo hacen continuamente, afirmaciones éstas basadas en los resultados de las entrevistas. Esta diferencia se debe precisamente a la esencia de lo que son las farmacias de autoservicio, ya que según León (1997), la estrategia de supervivencia en este tipo de negocios es la eficiencia en la estructura de costos, la multiplicidad de ofertas para atrapar a los consumidores, estar dispuestos a trabajar corrido y más de las ocho horas reglamentarias, mantener inventarios bajos de alta rotación y el ofrecimiento de servicios y productos conexos a la actividad farmacéutica, cosas que día a día van cambiando y mejorando para superar a su competencia.

Entretanto, el tiempo de respuesta tanto para farmacias tradicionales como de autoservicio es catalogado por los clientes como bueno; sin embargo, cada tipo de farmacia tiene su razón particular: las farmacias tradicionales tienen una cantidad de empleados mínima ya que el auge de clientes es bajo en casi todo su horario de trabajo mientras que las farmacias de autoservicio, por ser más grandes, ofrecen multiplicidad de servicios y poseen además una mayor demanda de clientes, tienen una nómina de empleados superior, razones por las cuales, al requerir ayuda algún cliente, siempre encuentran al menos un empleado disponible para atender sus necesidades.

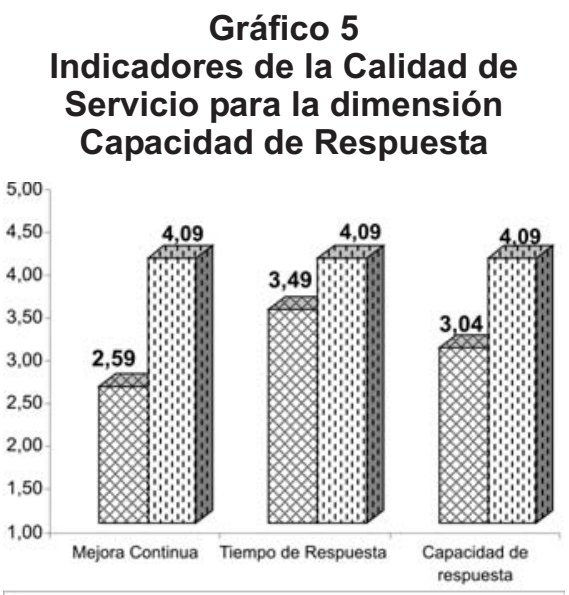

QFarmacias Tradicionales $\square$ Farmacias de Autoservicio

Fuente: Elaboración propia. Datos obtenidos a partir de las encuestas aplicadas (2009).

\section{Calidad de servicio en las farmacias tradicionales y de autoservicio de Maracaibo}

En el Gráfico 6 puede visualizarse la puntuación obtenida en cada una de las dimensiones que se establecieron para medir la calidad del servicio que ofrecen las diferentes modalidades de farmacia en el municipio Maracaibo; en la misma se evidencia que las farmacias de autoservicio alcanzaron mayor valor en todas sus dimensiones, de acuerdo a lo emitido por los clientes a través del instrumento aplicado, en relación a la calidad de servicio percibida en las farmacias tradicionales.

Según la escala empleada en el estudio, en las farmacias tradicionales se suministra un servicio de mediana calidad mientras que en las de autoservicio el servicio es de alta calidad, como se observa en el Diagrama 1. La línea puntea- 


\section{Gráfico 6 \\ Comparación de las dimensiones de la Calidad de Servicio en farmacias tradicionales y de autoservicio}

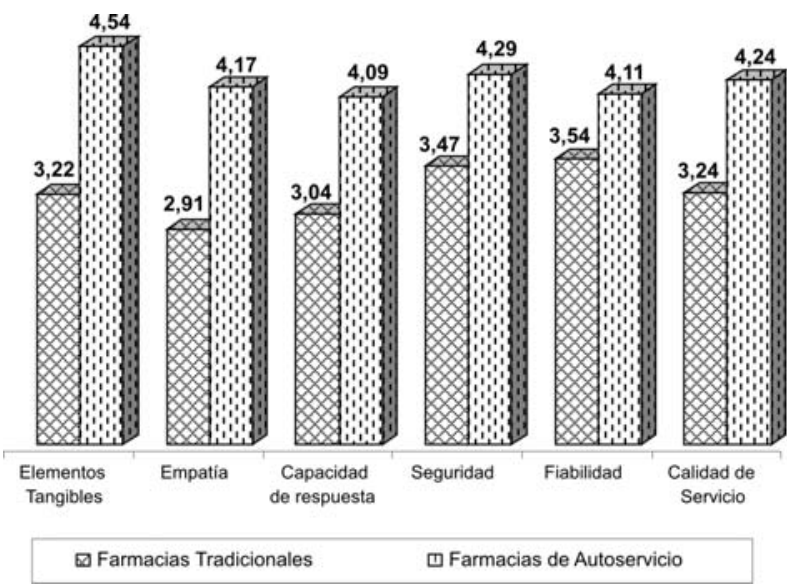

Fuente: Elaboración propia. Datos obtenidos a partir de las encuestas aplicadas (2009).

\section{Diagrama 1 \\ Diagrama radial comparativo de la Calidad de Servicio en farmacias tradicionales y de autoservicio}

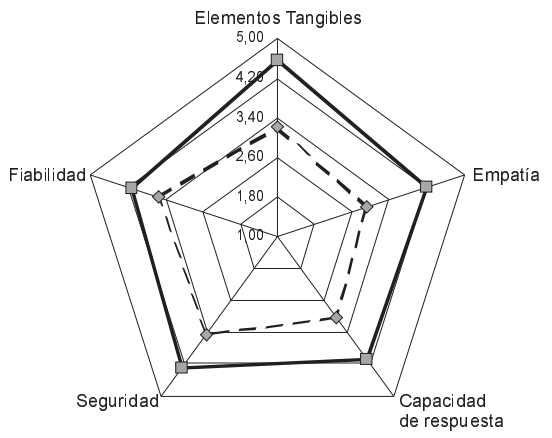

$-\diamond$ Farmacias Tradicionales $\multimap-$ Farmacias de Autoservicio

Fuente: Elaboración propia. Datos obtenidos a partir de las encuestas aplicadas (2009). da correspondiente a las farmacias tradicionales se encuentra en la tercera banda (de mediana calidad) mientras que la línea continua de las tradicionales se encuentra en la quinta banda de "alta calidad".

En el Gráfico 7 se observa la evaluación definitiva alcanzada por ambas modalidades de farmacia, en relación a la calidad de servicio que cada una de ellas ofrece a sus usuarios, luego del análisis de los resultados obtenidos por medio de las entrevistas realizadas a sus clientes.

Cuando un servicio es de buena calidad el cliente fija una imagen positiva de la farmacia y comúnmente va a repetir la experiencia. La calidad del servicio prestada en una organización repercute directamente en los beneficios finales de la misma y constituye uno de los medios por los cuales la empresa se diferencia en el mercado, logra crecimiento en los ne- 


\section{Gráfico 7 \\ Evaluación final de la calidad de servicio en las farmacias tradicionales y de autoservicio}

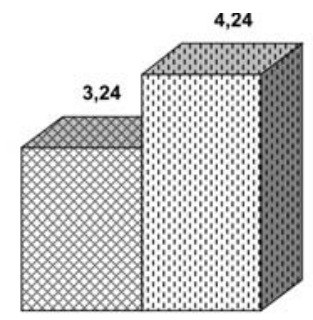

Calidad de Servicio

Q Farmacias Tradicionales $\square$ Farmacias de Autoservicio

Fuente: Elaboración propia. Datos obtenidos a partir de las encuestas aplicadas (2009).

gocios y obtiene resultados, alcanzando así las metas trazadas (Horovitz, 1994).

Según Albrecht y Laurence (1998), la calidad del servicio es esencial para las organizaciones, en virtud de que solo a través de ella, éstas pueden lograr el mantenimiento de la confianza de sus clientes actuales y la captación de clientes potenciales.

Horovitz (1994) señala que similar a un proceso de producción, la calidad del servicio se logra mediante un buen servicio la primera vez, logrando de esta forma la plena satisfacción del cliente, evitando la pérdida potencial de éstos, disminuyendo los costos y aumentando la productividad.

Por los basamentos anteriores se puede afirmar que las farmacias deben desarrollar y mantener altos niveles de calidad del servicio eliminando o disminuyendo sus debilidades en la medida de sus posibilidades, para así satisfacer las necesidades de los clientes que a ellas asisten, convirtiéndose de esta forma en el aliado de toda empresa de servicios. La calidad del servicio es entonces el sello distintivo entre una organización y otra. No importa si sus precios son más elevados dentro del segmento de mercado donde se encuentra. Lo importante para el cliente es saber que será tratado como un rey, y sus decisiones u opiniones serán tomadas en cuenta.

\section{Conclusión}

Producto del proceso de investigación emprendido para medir la calidad de servicio en las diferentes modalidades de farmacia del municipio Maracaibo, que permitieran comparar una con la otra, se emiten los siguientes resultados:

- Inicialmente se identificaron los indicadores adecuados para medir la calidad del servicio prestado en las farmacias, agrupándolos a su vez por dimensiones, a saber: elementos tangibles, fiabilidad, seguridad, empatía y capacidad de respuesta. De acuerdo con dichas dimensiones y según los indicadores tomados, la calidad del servicio fue medida a través de la adaptación del instrumento SERVPERF a las necesidades del estudio.

- De acuerdo a la percepción de la calidad de servicio de los clientes, las farmacias tradicionales obtuvieron rangos enmarcados desde la baja calidad de servicio hasta la buena calidad; asimismo, las farmacias de autoservicio se encontraron en los rangos de buena hasta alta calidad del servicio. 
- Basados en los resultados de la investigación, la calidad del servicio en las farmacias tradicionales del municipio Maracaibo es catalogada de mediana calidad por el $45 \%$ de los entrevistados; mientras que en las farmacias de autoservicio, fueron consideradas como establecimientos que brindan una alta calidad de servicio al cliente, según lo afirman el $55 \%$ de los entrevistados.

- Al destacar las debilidades de las farmacias tradicionales, resaltan los indicadores concernientes a la poca iluminación existente en el local, la expiración de los equipos utilizados o la nula utilización de los mismos, la apariencia poco acorde del personal, la ausencia de un farmaceuta (consejo farmacéutico), la inexistencia de actividades extras y servicios adicionales, y el trato neutral de los empleados como factores de mayor incidencia. Sin embargo, estas farmacias poseen un indicador a favor, que es el precio de venta de sus productos (básicamente medicamentos), ventaja altamente competitiva, la cual les ha ayudado a mantenerse en el mercado;

- Por su parte, las farmacias de autoservicio tienen que adicionar a sus productos un valor agregado por el servicio que prestan y la manutención de sus locales, costo que para las farmacias tradicionales es mínimo, convirtiéndose en una ventaja, ya que para los clientes que buscan precios más bajos, este tipo de farmacias son las más acordes.
- Las farmacias de autoservicio, por su parte le brindan al cliente más variedad de productos para adquirir en sus locales, cambiando el concepto antiguo de lo que es una farmacia y desplazando de forma avasallante a las conocidas "boticas de la esquina", ya que en la actualidad los comercios con bondades tecnológicas, así como también el denominado "autoservicio", constituyen las primeras opciones para la mayoría de las personas a la hora de conseguir medicamentos de primera necesidad. Sin embargo, algunas farmacias tradicionales tratan de mantenerse con su concepto de boticas y muchas personas las apoyan.

\section{Referencias bibliográfícas}

Albrecht, Karl; Bradford, Lawrence J. (1998). La Excelencia en el Servicio: ¡Conozca y comprenda a sus clientes! 1era. Edición. 3R Editores LTD. Colombia.

Bardera, Paula (2008). Ciencia y tecnología se dan la mano en Cenfarte. En: Revista PC World España. http://www.idg.es/ pcworldtech/Ciencia-y-tecnologia-sedan-la-mano-en-Cenfarte-/art193083 -pymes.htm (2009, enero 24)

Berry, Thomas (1992). Cómo gerenciar la transformación hacia la calidad total. Editorial McGraw Hill de Management. Colombia.

Cobra, Marcos (2000). Marketing de Servicios. 2da. Edición. Editorial McGraw Hill. Colombia.

Díaz, Paola (2008). El servicio al cliente y sus beneficios. http://www.miclienteyyo. com.ar/el-servicio-al-cliente-y-susbeneficios/ (2009, enero 28). 
Fernández, Alfonso. (2000). Calidad en las empresas de servicios. Editorial Instituto de fomento regional de Asturias. España

Franco, Lilia. (2002). La hora de los servicios. En: Revista Producto. Agosto 2002, $N^{\circ}$ 227. http://www.producto. com.ve/ 227/notas/portada8-12.html (2008, febrero 5).

Grupo EditorialProducto. (1998). Monte su farmacia. En: Revista Dinero. http:// www.dinero.com.ve/monte/farmacia. html (2009, enero 24).

Hernández, Rosa M.; Muñoz, Pablo A.; Santos, Libia (2006). Calidad objetiva y su relación con la formación y la satisfacción del empresario: El caso de los alojamientos rurales españoles. En: Documentos de Trabajo "Nuevas Tendencias en Dirección de Empresas". España. http://www3.uva.es lempresa/uploads/dt_13_06.pdf. (2009, Septiembre 27).

Horovitz, Jacques. (1994). La Calidad del Servicio a la Conquista del Cliente. Editorial McGraw Hill. Madrid.

Kotler, Philip; Armstrong, Gary (1998). Mercadotecnia. Editorial Prentice Hall. México.

McCarthy, Jerome; Perreault, William D. (1999). Marketing, un enfoque global. $13^{\mathrm{a}}$ edición. McGraw Hill. México.

Monroy, Martín. (2006) La actividad comercial. http://editorial.cda.ulpgc.es/instalacion/7_OPTATIVAS/LAU/LAU2 _ tipologia/lau23.htm (2009, enero 24).

Orjuela, Ingrid. (2003). El negocio de turno. Farmacias. En: Revista Producto Septiembre $2003 \mathrm{~N}^{\circ}$ 238. http://www. producto.com.ve/238/notas/portada. html (2008, febrero 5)

Parasuraman, A.; Zeithalm, Valarie; Berry, Leonard (1991). Calidad Total en la gestión de Servicios. Editorial Díaz de Santos. España.
Robledo, Marco A. (2004). Modelos de medición de la calidad de servicio: su aplicación a empresas de transporte aéreo. En: Cuadernos de economía y dirección de la empresa, ISSN 1138-5758, № 18, 2004, Págs. 147172. http://dialnet.unirioja.es/servlet/ articulo?codigo $=1143677 . \quad$ (2009, Septiembre 11).

Rojas, Héctor (1997). Mejoramiento de la calidad de servicios mediante la orientación al cliente y el compromiso de la empresa http://www.monografias.com/trabajos15/calidadserv/ calidad-serv.shtml (2008, octubre 21).

Romero, Gerardo (2006). Principios y valores éticos en las organizaciones bancarias respecto a la calidad del servicio y atención al cliente. En: Revista Venezolana de Gerencia. Octubre-Diciembre, Año 11, vol. 11, N 036. LUZ, Maracaibo, Venezuela. Pp. 638-651. http://www.serbi.luz.edu.ve/scielo.ph p? pid=S1315-99842006012000008 \& script (2009, enero 24).

Setó, Dolors (2004). De la calidad de servicio a la fidelidad del cliente. Editorial ESIC. Madrid, España. http://books. google.co.ve/books?id=9Nk8s WMjo BcC (2008, octubre 21).

Wharton School. (2005). La frenética expansión de las cadenas de farmacias por Latinoamérica. http://wharton. universia.net/index.cfm?fa=viewArti cle\&id=943 (2009, enero 29).

Zubillaga, Rafael (2008). 90 años sirviendo a Venezuela bajo techos azules. En Bussiness on line Venezuela. http://www.bvonline.com.ve/295/sp cprofile.html (2009, enero 28).

Zeithaml, Valarie; Bitner, Mary Jo (2002). Marketing de servicios. 2da Edición. McGraw Hill. México. 